${ }^{1}$ Centro de Bioética, Facultad de Medicina Clínica Alemana Universidad del Desarrollo. Santiago, Chile. ${ }^{2}$ Comité de Ética en Investigación, Hospital Sótero del Río. Santiago, Chile. ${ }^{3}$ Facultad de Medicina, Universidad Diego Portales. Santiago, Chile. aFonoaudióloga;

Fuente de apoyo financiero: No existe. Este trabajo no obtuvo financiamiento.

Recibido el 5 de septiembre de 2016, aceptado el 8 enero de 2017.

Correspondencia: María Bernardita Portales Velasco Las Condes 12.438, Lo Barnechea bportales@udd.cl

\section{Formas de Revisión Ética de Proyectos de Investigación Biomédica}

\author{
MARÍA BERNARDITA PORTALES ${ }^{1, A}$, PATRICIO MICHAUD², \\ SOFÍA P. SALAS ${ }^{3}$, JUAN PABLO BECA ${ }^{1}$
}

\section{Differentiated review of biomedical research projects by ethics committees}

Ten years after the approval of the Chilean bill that regulates scientific research in humans (Law No 20.120), and considering the current status of accreditation and training of many Research Ethics Committee (REC), it is necessary to analyze their performance. We analyzed the Chilean experience with REC aiming to propose a differential type of review, considering the risks to research participants. To improve the quality of the review and the efficiency of these committees, we propose to differentiate the revisions depending on the type of project, its methods and its risks. Initially, the types of review should be classified as exempt from review, expedited review and full review by the committee. In this proposal the type of review is confirmed or can be modified by a designated member of the committee after an initial review of the project. Thus, the deliberation and review times of the committee could be optimized avoiding delays in their revision.

(Rev Med Chile 2017; 145: 386-392)

Key words: Bioethics; Ethics Committees, Research; Ethics, Institutional; Ethics, Research.

\section{L} a investigación biomédica ha aumentado en forma progresiva en Chile en los últimos años, tal como lo muestra una revisión en PubMed de artículos con la palabra Chile AND human, que incrementan desde 592 artículos publicados en el año 1995 a 1900 en el año 2015. Esto ha llevado a una mayor demanda de trabajo de los Comités Ético Científicos (CEC). Los primeros CEC nacieron en Chile en los años 80 y actualmente la mayoría se encuentra en proceso de acreditación por el Ministerio de Salud ${ }^{1}$. El principal objetivo de los CEC es la protección de los sujetos participantes en una investigación, contribuyendo al desarrollo de investigación de excelencia ética y científica, según lo señalan documentos como la Declaración de Helsinki $2013^{2}$ y las Pautas CIOMS 3 .

En la investigación biomédica, existen diversos tipos de proyectos que se diferencian según su fuente de financiamiento (fondos académicos, de la industria farmacéutica o fondos concursables); según los sitios de su realización (multicéntricos o en un solo centro); y también según si hay o no intervención con los sujetos participantes. Esta diferenciación en proyectos con o sin intervención o interacción permite una primera aproximación a los posibles riesgos y beneficios de una investigación, guiando así a revisiones de distinta profundidad.

Después de 10 años de promulgada la ley $\mathrm{N}^{\circ} 20.120$ que regula la investigación en Chile ${ }^{4}$, resulta oportuno realizar una mirada crítica a los tipos de revisión de proyectos de investigación biomédica, para optimizar el tiempo y mejorar la calidad de la revisión ética de los protocolos. Con esta finalidad, se recogió la historia de los CEC en Chile, se revisaron algunas formas de trabajos y experiencias para, finalmente, proponer criterios 
y formas de revisión de acuerdo a tipos de proyectos, sus métodos y sus riesgos. Por motivos de espacio, se ha dejado fuera de este análisis otros aspectos importantes relacionados con el trabajo de los CEC, como son aquellos relacionados con formación continua y contribución a mejorar los estándares de la ética de los investigadores.

\section{Historia de la revisión ética en investigación y de los CEC en Chile}

Antes del Siglo XX, los aspectos éticos de la investigación biomédica se auto-regulaban en base a la conciencia del investigador y por sus códigos de conducta profesional. Hacia comienzos del siglo pasado, ya existía amplia experiencia respecto de los problemas éticos de la investigación científica en seres humanos, incluyendo nociones de voluntariedad de participación y balance de riesgos versus beneficios ${ }^{5}$. A partir de la segunda mitad del siglo XX, por diversas razones entre las que se encuentran algunas relacionadas con conductas inaceptables de algunos investigadores, se hizo evidente que la investigación en sujetos humanos requería una revisión por pares, puesto que la sola autoregulación del investigador resultaba insuficiente para proteger adecuadamente a los sujetos de investigación ${ }^{5,6}$.

En Chile, los comités de ética de la investigación se crearon en las principales instituciones de educación superior, fundamentalmente en sus facultades de Medicina. Estas instituciones impusieron procesos de revisión por pares de los aspectos éticos para todo proyecto de investigación biomédica. La Escuela de Medicina de la Pontificia Universidad Católica de Chile creó la "Comisión de Investigación Científica"en 1976 y al año siguiente redactó normas éticas para la investigación clínica, las cuales establecían la obligatoriedad de revisión científica y ética de todo proyecto de investigación en seres humanos por parte de la Comisión ${ }^{7}$. Al poco tiempo, la Universidad de Chile creó la "Comisión de Ética, Cultura e Historia", la que en el año 2001 dio origen al Comité de Bioética sobre Investigación en Seres Humanos, que tenía como misión revisar los proyectos de investigación que realizaba la Facultad de Medicina, para así proteger los derechos, la seguridad y el bienestar de los sujetos de investigación ${ }^{8}$. En el sector público de salud, en 1996 se creó el Comité Ético Científico del Hospital Sótero del Río, el cual en el año 2001 pasó a ser el Comité Ético Científico del Servicio de Salud Metropolitano Sur Oriente ${ }^{9}$, y en esa misma época comenzó a funcionar el Comité de Ética de la Investigación del Hospital del Salvador.

En el año 1991 CONICYT estableció dentro de los requisitos de postulación el contar con una aprobación previa por parte del Comité de Ética Institucional y, a partir del Concurso del año 1993 se exigió la aprobación por parte de la Institución Patrocinante ${ }^{10}$. En el año 2005 se constituyó el Comité Asesor de Bioética de FONDECYT, cuyo fin principal era reforzar los aspectos bioéticos concernientes a las actividades científicas patrocinadas por la institución, comité que actualmente sigue en funciones ${ }^{11}$.

Desde el punto de vista jurídico, en el año 2000 el MINSAL estableció una normativa que creó los Comités de Evaluación Ético Científicos con la finalidad de informar sobre las investigaciones en humanos con uso de medicamentos no registrados en el país ${ }^{12}$. En el año 2001, la Norma Técnica $\mathrm{N}^{\circ}$ 57 que regula el uso de productos farmacéuticos en ensayos clínicos, estableció la necesidad de revisión de protocolos de investigación por un Comité de Evaluación Ético-Científica. Este comité debía evaluar "los aspectos científico-técnicos y éticos del protocolo de investigación, de los investigadores, del sitio donde se realice la investigación y de la institución patrocinadora" y establecía que se podía designar un Comité de Evaluación Ético-Científico de Referencia cuando los Servicios de Salud no contaran con uno propio ${ }^{13}$. En el año 2006 fue promulgada la Ley 20.120 que estableció, por primera vez con rango legal, el requisito de aprobación ética de los proyectos de investigación en o con humanos ${ }^{4}$. Posteriormente, en el año 2010 se publicó el Decreto No 114 con el Reglamento respectivo, cuyo Párrafo Tercero está dedicado a los Comités Ético Científicos, especificando qué son, cómo debiesen estar constituidos, y sus atribuciones ${ }^{14}$.

En el Decreto 30 que modifica el Reglamento sobre la ley 20.120, se establece que "Toda investigación científica en seres humanos a realizarse en el país sólo podrá llevarse a cabo si cuenta con una revisión e informe favorable de un Comité Ético Científico acreditado por la Autoridad Sanitaria e independiente del equipo de investigación. En los casos de los estudios multicéntricos, la revisión científica y ética se realizará por un solo comité 
acreditado, debiendo ser complementada por una evaluación local relacionada con los investigadores e instituciones que participan en ese nivel para verificar la factibilidad del estudio en sus comunidades, incluyendo la infraestructura, el nivel de capacitación y las consideraciones éticas locales significativas"15.

Actualmente cada CEC tiene un reglamento interno que define sus propias formas de trabajo de acuerdo a las condiciones locales, frecuencia de reuniones y la posibilidad de revisión expedita de protocolos de investigación. Al 11 de noviembre de 2016, existen 34 Comités acreditados ${ }^{1}, 29$ de los cuales lo están para revisar ensayos clínicos. El número de integrantes varía entre 5 (mínimo que establece la ley) a 24; los más numerosos corresponden a Comités universitarios. De acuerdo a la información obtenida por los autores, quienes participan en tres Comités diferentes, los CEC han trabajado cada día de manera más organizada, aunque sólo algunos de ellos establecen en sus reglamentos formas diferentes de revisión según tipo de proyectos.

\section{Criterios de revisión y tipos de investigación}

Si bien cada CEC puede establecer sus guías para la revisión de proyectos de investigación, parece razonable precisar distintos criterios en la revisión ética de un proyecto de investigación biomédica, de acuerdo a lo establecido en la Norma Técnica 151 referida a los estándares de acreditación de los $\mathrm{CEC}^{16}$, considerando los requisitos propuestos por Ezequiel Emanuel ${ }^{17}$. En el presente artículo se sugiere que las revisiones éticas se concentren en los siguientes tres criterios, sin establecer un orden jerárquico entre los mismos:

\section{1) Protección del sujeto que participa en la in-} vestigación, incluyendo aspectos relacionados con balance riesgo beneficio y protección de la confidencialidad de los participantes:

1.1. Riesgos a los que se somete el sujeto por participar en la investigación, lo que implica que el investigador es el principal protector del sujeto participante. Este criterio involucra que se evalúe:

a) Relación riesgo-beneficio favorable y minimización de los riesgos. Se sugiere máxima rigurosidad en la revisión de este criterio en los estudios clínicos fase I y fase II. b) Compensación, mediante seguros o garantías, de los eventos adversos que pudieran preverse o que, sin ser previsibles, causen daño a las personas participantes que se relacionen directamente con su participación en el estudio. Estos aspectos han quedado también establecidos en la Ley 20.850 de protección financiera para diagnósticos y tratamientos de alto costo, cuyos Artículos 111E y F abordan el tema de la responsabilidad por daños producidos con ocasión de la investigación y la obligación por parte de los titulares de las autorizaciones para uso provisional para fines de investigación de productos farmacéuticos o de uso médico de contar con una póliza de seguro de responsabilidad civil ${ }^{18}$.

c) Evaluar si existe algún mecanismo para informar a los sujetos participantes en caso de obtención de datos o información nueva acerca de los riesgos y beneficios de las intervenciones utilizadas o de hallazgos incidentales, y qué hará el investigador en esas situaciones.

d) Vigilar cuidadosamente el bienestar del sujeto a lo largo de la investigación. Este aspecto considera llevar a cabo el seguimiento de los estudios y que el investigador responsable haga llegar la documentación respectiva al CEC.

e) En el caso de intervenciones terapéuticas, se debe establecer la seguridad de que los participantes podrán tener acceso a la continuidad del tratamiento demostrado como eficaz, incluyendo la rama que recibió placebo.

1.2. Confidencialidad de los participantes: el comité debe conocer la forma de protección de la intimidad de los sujetos y el resguardo de la confidencialidad de los datos y registros de las personas que participan en investigación, tal como señala la ley de protección de la vida privada $^{19}$.

\section{2) Garantizar la voluntariedad en la participa-} ción: el sujeto es un agente activo en la investigación, por lo tanto, en el protocolo deberá quedar clara la seguridad y forma de respeto de la voluntariedad de la participación de los sujetos, quién solicitará y cómo se llevará a cabo el proceso de consentimiento informado. Este aspecto abarca también:

a) Protección especial de grupos vulnerables, como lo son inmigrantes, población penal, 
niños, adultos mayores, personas con discapacidad mental y beneficiarios sin acceso a otro tipo de atención, entre otros.

b) Revisión de los documentos que entregan información y en los que se registra el consentimiento informado y asentimiento en caso de menores de edad, a lo que se suma el material de apoyo como avisos de reclutamiento de participantes. De particular relevancia es verificar que los documentos sean comprensibles de acuerdo al nivel socio-cultural y literacidad de los sujetos. En estos documentos debe quedar claro el hecho de permitir al sujeto cambiar de opinión y retirarse de la investigación sin sanción alguna. En proyectos que incluyan pago, incentivos o regalos por participar, el CEC debe asegurar que estos aportes no limiten la libertad de decidir, si estas retribuciones fuesen desproporcionados para la realidad local.

c) Proceso de consentimiento informado: quién, cómo y cuando va a solicitar el consentimiento y cómo va a entregar la información, lo cual cobra mayor relevancia en sujetos o poblaciones vulnerables.

d) Selección equitativa de las personas que participan en una investigación: de acuerdo a lo planteado por Emanuel, este criterio tiene cuatro aspectos a considerar: "asegurar que se seleccionen grupos específicos de sujetos por razones relacionadas con las interrogantes científicas incluidas en la investigación; a todos los grupos se les debe ofrecer la oportunidad de participar en la investigación a menos que existan razones científicas o de riesgo que restringiesen su elegibilidad; los sujetos que se reclutan están en condiciones de beneficiarse si la investigación proporciona un resultado positivo (como podría ser un nuevo tratamiento) y, garantía de una razón de riesgo-beneficio apropiada" 20 .

\section{3) Asegurar la Validez Científica y el Valor Social:}

el comité deberá asegurarse que el estudio esté metodológicamente bien diseñado y que pueda cumplir con los objetivos propuestos. Dentro de este criterio, se puede incluir también que la investigación cuente con investigadores idóneos, con la experiencia y calificación profesional acorde con el nivel de complejidad de la investigación y que no exista conflicto de intereses de los investigadores. Tal como lo propone Emanuel ${ }^{20}$, una investigación tiene valor si cumple con algunas de estas características:

a) La investigación evalúa una intervención diagnóstica o terapéutica que podría mejorar la salud de la población.

b) Es un estudio preliminar (etiológico, patofisiológico o epidemiológico) orientado a desarrollar dichas intervenciones.

c) Prueba una hipótesis que puede generar importante conocimiento sobre la estructura o función de los sistemas biológicos humanos, aunque no tenga aplicación inmediata.

d) La investigación debería tener impacto en la comunidad a la que pertenecen los sujetos que participan en ella.

e) Debe existir algún mecanismo para informar, una vez concluido el estudio, a los participantes sobre los resultados y lo que se aprendió de la investigación clínica.

a) Identificación de los intereses de la población local en proyectos internacionales, asegurando que los participantes recibirán beneficios razonables.

\section{Tipos de investigación y de revisión}

De acuerdo al tipo de investigación y la evaluación riesgo - beneficio para los participantes, deberían distinguirse dos grandes categorías básicas en los proyectos de investigación biomédica: con o sin intervención en los sujetos. Los tipos de investigación de cada una de estas categorías se detallan en la Tabla 1.

Se propone que la revisión sea diferenciada según una primera aproximación de los riesgos a los que se expone el sujeto. En los Estándares de Acreditación de los CEC y en las respectivas Pautas de Autoevaluación se establece que se requiere que los comités tengan criterios para revisión expedita y eximición de revisión, que deben estar indicados en el reglamento, definiendo qué tipo de proyecto será sometido a este tipo de revisión ${ }^{16,21}$. Se entiende que, en general, en aquellos que hay intervención o interacción con el sujeto, existe riesgo potencial de daño. Tal como lo señala el Código de Regulaciones Federales americano (CFR de sus siglas en inglés), la intervención puede corresponder a procedimientos físicos requeridos para la recolección de datos (por ejemplo, punción venosa) o manipulaciones al sujeto o a su ambiente 
Tabla 1. Tipos de Proyectos de Investigación Biomédica con intervención y sin intervención

\begin{tabular}{|ll|}
\hline Proyectos con intervención & Proyectos sin intervención \\
\hline - Fármacos & - Uso de muestras biológicas obtenidas por indicación clínica \\
\hline - Vacunas & - Uso de base de datos clínicos \\
\hline - Dispositivos & - Uso de registros de imágenes \\
- Terapia génica & - Uso de muestras de Biobancos \\
\hline - Innovación quirúrgica & - Uso de fichas clínicas \\
- Procedimientos diagnósticos & - Sociales \\
\hline - Sociales & \\
\hline - Psicológicos/psicoterapéuticos & \\
\hline - Obtención muestras biológicas para investigación & \\
\hline
\end{tabular}

con propósitos de investigación; la interacción, en cambio, incluye comunicación o contacto interpersonal entre el investigador y el sujeto de investigación ${ }^{22}$.

Adaptando a la realidad chilena lo planteado por Emanuel ${ }^{20}$ y lo que señala el CFR, se proponen tres alternativas de tipos de revisión: eximido de revisión, revisión expedita y revisión por el comité en pleno:

\section{Proyectos eximidos de revisión}

Son aquellos que el Comité exime de ser revisados por tratarse de investigaciones sin interacción con los participantes. Esta categoría debe ser decidida por el Presidente del CEC o por la persona designada para estos fines. Un ejemplo de este tipo de investigación es aquella que involucra la colección o estudio de datos ya existentes, documentos, registros, especímenes patológicos o diagnósticos, si esas fuentes están disponibles de manera pública o si la información es recogida por el investigador de una manera anonimizada; así como proyectos que evalúan el beneficio de ciertos programas públicos o prácticas educacionales habituales, sin que exista intervención ${ }^{23}$. También se pueden incluir proyectos que no corresponden a una investigación propiamente tal - como evaluaciones de procesos locales o de calidad - que no involucran mayormente a las personas, que no tienen por objeto ser un aporte al conocimiento, o donde no se plantea una hipótesis o pregunta a contestar. El CEC certificará la eximición y esta decisión deberá quedar en el acta y registro correspondiente.

\section{Los proyectos de revisión expedita}

Investigaciones con riesgos mínimos. Tal como lo señalan las Pautas CIOMS 2016, el estándar del riesgo mínimo se refiere a los riesgos inherentes a aquellas actividades cotidianas a las cuales habitualmente se ve expuesta una persona sana (como podría ser caminar por la calle o hacer ejercicio moderado) o durante el desarrollo de exámenes físicos o psicológicos de rutina, incluyendo la visita al servicio de salud ${ }^{24}$. Este tipo de proyectos requieren ser revisados por uno o dos miembros del comité designados para esta función, dentro de los que, generalmente estará el presidente o el secretario del CEC. En esta categoría podrán entrar también la revisión de enmiendas al protocolo y cambios en los documentos de consentimiento informado, siempre que no impliquen cambios en la validez científica del estudio o modificación del balance riesgo/beneficio inicial. De acuerdo a los riesgos pesquisados en una primera evaluación o a la experiencia del CEC, esta revisión expedita podría pasar a una revisión del protocolo por el Comité en pleno.

\section{Los proyectos de revisión por el comité en pleno}

Investigaciones con intervenciones que impliquen riesgos mayores al mínimo, como son los estudios de fármacos, vacunas o dispositivos, cualquiera sea su fase, o si se busca probar un nuevo uso para un fármaco. Estos proyectos requieren una revisión exhaustiva realizada por el comité en pleno, tanto del protocolo como de la documentación del Consentimiento Informado. 
Tabla 2. Resumen de los tipos de revisión según las características del estudio

\begin{tabular}{|c|c|c|}
\hline Tipo de revisión & Definición & Ejemplos \\
\hline Eximidos & $\begin{array}{l}\text { Proyectos que el CEC exime porque no impli- } \\
\text { can riesgos para los sujetos participantes en la } \\
\text { investigación y que no requieren de consenti- } \\
\text { miento informado } \\
\text { Proyectos sin participación de sujetos de inves- } \\
\text { tigación o que no usan información personal } \\
\text { El CEC emitirá un certificado de eximición }\end{array}$ & $\begin{array}{l}\text { Análisis secundario de base de datos anonimizadas } \\
\text { o cuando la privacidad está debidamente asegurada } \\
\text { Reporte de casos con garantía de respeto a la priva- } \\
\text { cidad y confidencialidad de la información } \\
\text { Revisiones de políticas públicas y reglamentación } \\
\text { Revisiones de base de datos de uso público (por } \\
\text { ejemplo, datos del INE) }\end{array}$ \\
\hline Expedito & $\begin{array}{l}\text { Proyectos que tienen riesgo mínimo, o enmien- } \\
\text { das al protocolo que no impliquen cambios en } \\
\text { la validez científica. } \\
\text { Son investigaciones que serán revisadas por } \\
\text { uno o dos miembros del CEC }\end{array}$ & $\begin{array}{l}\text { Proyectos que incluyen uso de muestras de material } \\
\text { biológico, imágenes o muestras de biobancos que } \\
\text { han sido obtenidas por indicación clínica }\end{array}$ \\
\hline Comité en pleno & $\begin{array}{l}\text { Proyectos con riesgos mayores al mínimo para } \\
\text { los sujetos participantes }\end{array}$ & $\begin{array}{l}\text { Estudios clínicos con fármacos, vacunas, dispositi- } \\
\text { vos, innovación quirúrgica. } \\
\text { Proyectos con métodos diagnósticos invasivos } \\
\text { Proyectos con intervención social } \\
\text { Proyectos con intervención psicológica/psicotera- } \\
\text { péuticos }\end{array}$ \\
\hline
\end{tabular}

Un resumen de los tipos de revisión y algunos ejemplos, según las características del estudio se muestran en la Tabla 2.

Cada Comité designará uno de sus miembros como la persona encargada de decidir el tipo de revisión al que será sometido el proyecto, según una primera aproximación de sus riesgos. De acuerdo a cada realidad, o ante cualquier duda en el protocolo, los Comités pueden invitar al investigador responsable a exponer el proyecto en una sesión del Comité. Todo proyecto revisado, incluso los eximidos, deben quedar en actas del CEC y deben contar con su respectiva carta de certificación, ya sea de eximición, aprobación, pendiente para modificaciones, o de rechazo. Asimismo, los criterios de los tipos de revisión deberían quedar consignados en las Pautas de funcionamiento que cada comité haya establecido en su reglamentación.

\section{Conclusión}

Desde la promulgación de las leyes relacionadas con investigación en seres humanos y que hacen obligatorio su revisión por parte de CEC acreditados, fijando plazos y procedimientos, sumado al progresivo aumento de la investigación en distintos centros académicos, se ha incrementado la demanda de trabajo para los CEC, con sus limitados recursos humanos y materiales.

La propuesta de poder diferenciar los tipos de revisión en eximidos, expeditos y revisión en el Comité en pleno, constituye una forma para que sin descuidar la debida protección a los sujetos participantes, se pueda optimizar el trabajo del Comité, permitiendo que se destine mayor tiempo de revisión a aquellos proyectos con mayor riesgo potencial para los sujetos, como son las investigaciones con intervención.

\section{Referencias}

1. Comités de Etica Científicos Acreditados, MINSAL, Chile. Disponible en http://ish.redsalud.gob.cl/?page_id=315. Fecha de acceso 11/11/2016.

2. World Medical Association Declaration of Helsinki: ethical principles for medical research involving human subjects. JAMA 2013; 310(20): 2191-4.

3. Pautas éticas internacionales para la investigación biomédica en seres humanos. Preparadas por el Consejo de Organizaciones Internacionales de las Ciencias Médicas (CIOMS) en colaboración con la Organización Mundial de la Salud. Ginebra, 2002. Disponible en http://www. cioms.ch/publications/guidelines/pautas_eticas_internacionales.htm. (Consultado el 3 de marzo de 2016).

4. Ley 20.120. Sobre la investigación científica en el ser 
humano, su genoma, y prohibe la clonación humana. Ministerio de Salud de Chile, promulgada el 7 septiembre 2006. Disponible en http://bcn.cl/1m19j. CC (Consultado el 3 de marzo de 2016).

5. Schneider WH The establishment of Institutional Review Boards in the U.S. Background history. Disponible en http://www.iupui.edu/ histwhs/G504.dir/irbhist. html. (Consultado el 26 de enero de 2016).

6. Beecher HK. Ethics and clinical research. N Engl J Med 1966; 274(24): 1354-60.

7. Salas SP. Estructura y función de los comités de ética de la investigación clínica. Cuadernos del Programa Regional de Bioética 1996; 3: 89-105.

8. Facultad de Medicina Universidad de Chile. Resolución No 3922 del 19 noviembre de 2013. Modifica resolución que constituyó el Comité de Bioética sobre Investigación en Seres Humanos de la Facultad de Medicina, sustituye su denominación y establece normas sobre su composición, funciones, atribuciones y funcionamiento. Disponible en http://ceish.med.uchile.cl/documentos_ceish. html. (Consultado el 3 de marzo de 2016).

9. Comité Ético Cientifico del SSMSO. Disponible en http://ssmetroso.redsalud.gob.cl/?p=1791. (Consultado el 3 de marzo de 2016).

10. Velasco P. Aspectos Bioéticos de la investigación científica en proyectos Conicyt/Fondecyt. Libro 1 Bioética e investigación con seres humanos y en animales 1 Taller de Bioética, organizado por el Comité Asesor de Bioética de CONICYT 2005; 129: 33.

11. FONDECYT. Bases concurso nacional regular 2005.Disponible en http://www.conicyt.cl/fondecyt/2005/05/19/ concurso-nacional-regular-2005/. (Consultado el 26 de enero de 2016).

12. Santos M, Michaud P, Méndez G, Téllez R, Sotomayor A, Winkler M, et al. Funcionamiento de Comités de Bioética de Investigación en seres humanos: Experiencia nacional en el área de FONDECYT de Ciencias Biomédicas. Libro 5 Ética de los Comités de Ética y Bioética en Investigación Científica, Biomédica y Social Editado por la Comisión Nacional de Investigación Científica y Tecnológica, CONICYT, Ministerio de Educación, Gobierno de Chile 2010; 5to Taller de Bioética: 48-57.

13. Norma Técnica No 57: Regulación de la ejecución de ensayos clínicos que utilizan productos farmacéuticos en seres humanos. Departamento Programa de Personas, Ministerio de Salud, Chile, 2001. Disponible en http:// www.ispch.cl/ensayos-clinicos. (Consultado el 14 de junio de 2006).

14. Decreto 114. Aprueba Reglamento de la Ley No 20.120, sobre la investigación científica en el ser humano, su genoma, y prohíbe la clonación humana. Fecha promul- gación 22 de noviembre de 2010. Disponible en http:// bcn.cl/1mh6g. (Consultado el 3 de marzo de 2016).

15. Decreto 30. Modifica decreto $\mathrm{N}^{\circ} 114, \mathrm{DE} 2010$, que aprueba Reglamento de la Ley N ${ }^{\circ} 20.120$, sobre la investigación científica en el ser humano, su genoma y prohibe la clonación humana. Disponible en http://bcn. cl/1x5ae. (Consultado el 3 de marzo de 2016).

16. Norma General Técnica No 151 sobre estándares de acreditación de los comités ético científicos. Ministerio de Salud, Chile. Resolución Exenta No 403 del 11 julio 2013. Disponible en http://web.minsal.cl/portal/url/ item/e52b95d04dafbcece04001016401571d.pdf. (Consultado el 28 de marzo de 2016).

17. Emanuel EJ. Reform of Clinical Research Regulations, Finally. N Engl J Med 2015; 373(24): 2296-9.

18. Ley 20.850. Crea un sistema de protección financiera para diagnósticos y tratamientos de alto costo y rinde homenaje póstumo a Don Luis Ricarte Soto Gallegos. Ministerio de Salud de Chile. Promulgada el 1 junio 2015. Disponible en http://bcn.cl/1v7lo. (Consultado el 29 de agosto de 2016).

19. Ley No 19.628. Sobre protección de la vida privada. Ministerio Secretaría General de la Presidencia. Promulgada el 18 de agosto de 1999. Disponible en http://bcn. cl/1uv2v. (Consultado el 29 de agosto de 2016).

20. Emanuel EJ, Wendler D, Grady C. What makes clinical research ethical? Jama 2000; 283(20): 2701-11.

21. Pautas de Autoevaluación para el proceso de acreditación de Comités Ético Científicos. Disponible en http:// ceish.med.uchile.cl/ley/CEC Pautas autoevaluacion.pdf. (Consultado el 7 de junio de 2016).

22. Code of Federal Regulations. Title 45 Public Welfare Department of Health and Human Services. Part 46 Protection of Human Subjects. 46.110 Expedited review procedures for certain kinds of research involving no more than minimal risk, and for minor changes in approved research. Vigente desde el 14 de julio 2009. Disponible en http://www.hhs.gov/ohrp/ regulations-and-policy/regulations/45-cfr-46/ - 46.110. (Consultado el 29 de agosto de 2016).

23. Code of Federal Regulations. Title 45 Public Welfare Department of Health and Human Services. Part 46 Protection of Human Subjects. 46.101 To what does this policy apply? Vigente desde el 14 de julio 2009. Disponible en http://www.hhs.gov/ohrp/regulations-and-policy/ regulations/45-cfr-46/ - 46.110. (Consultado el 29 de agosto de 2016).

24. International ethical guidelines for health-related research involving humans. Geneva, Switzerland: Council for International Organizations of Medical Sciences (CIOMS), 2016. 